\title{
Variación estacional en la composición de ensambles sublitorales de macroalgas asociadas al alga roja Gigartina skottsbergii Setchell \& Gardner, en el Estrecho de Magallanes, Chile
}

Seasonal variation in the composition of subtidal macroalgal assemblages associated with the red macroalga Gigartina skottsbergii Setchell \& Gardner, in the Strait of Magellan, Chile

Johanna Marambio ${ }^{1,2}$, Sebastián Rosenfeld ${ }^{1,2}$, Jaime Ojeda ${ }^{1}$, Andrés Mansilla ${ }^{1,2}$

\section{Resumen}

La creciente demanda de carragenanos por parte de la industria chilena y mundial ha provocado una fuerte presión extractiva sobre las praderas naturales de 'luga roja', Gigartina skottsbergii, desplazando el esfuerzo pesquero desde los $41^{\circ} \mathrm{S}$ hacia la zona más austral de Chile $\left(54-56^{\circ} \mathrm{S}\right)$. A pesar del conocimiento generado sobre la biología de G. skottsbergii, se desconocen las macroalgas asociadas a sus praderas naturales, por lo tanto no se sabe cómo afectan factores estresantes como: cambio climático, acidificación de los océanos, pesquería artesanal, entre otros, sobre los organismos asociados a praderas de G. skottsbergii. En este trabajo se evalúa estacionalmente la composición de las macroalgas asociadas a dos praderas ubicadas en el Estrecho de Magallanes. Los sitios de estudio corresponden a Santa María (Isla Tierra del Fuego) y Santa Ana (Península de Brunswick). Se identificaron un total de 31 especies (21 Rhodophyta, 7 Ochrophyta and 3 Chlorophyta). La riqueza promedio de especies por cuadrante, en Santa Ana presentó un aumento significativo durante el verano $(p<0,05)$, presentando la mayor riqueza en otoño $(4,2 \pm 0,3)$, mientras que, en Santa María no se observaron diferencias significativas entre las estaciones del año $(p>0,05)$. Los análisis multivariados arrojaron diferencias significativas en la composición de los ensambles de macroalgas entre las dos localidades, para cada estación del año $(p<0,05)$. Este trabajo entrega el primer inventario estacional de ensambles sublitorales de macroalgas en dos localidades del Estrecho de Magallanes.

Palabras claves: Subantártico, macroalgas, biodiversidad, estacional, sublitoral.

\section{Abstract}

The emergent demand for carrageenan by Chilean and global industries caused increasing extraction pressures on natural populations of 'red luga', Gigartina skottsbergii, displacing fishing effort from $41^{\circ} \mathrm{S}$ to the southernmost region of Chile (54-56 $\left.{ }^{\circ} \mathrm{S}\right)$.

Despite the knowledge generated in the biology of $G$. skottsbergii, unknown the flora associated with G. skottsbergii, therefore we don't know, the real impacts of: global warming, ocean acidification, extractive fishery, among others, in benthic community associated with $G$. skottsbergii. In this work we evaluate seasonal composition of macroalgae associated with two populations of $G$. skottsbergii in the Strait of Magellan. The study sites corresponded to Santa María (Tierra del Fuego Island) and Santa Ana (Brunswick Peninsula). We identified a total of 31 species (21 Rhodophyta, 7 Ochrophyta and 3 Chlorophyta). The species richness

Laboratorio de Macroalgas Antárticas y Subantárticas (LMAS), Universidad de Magallanes, Punta Arenas, Chile. $>$ johanna.marambio@yahoo.com, s86r7s5@gmail.com, jaimeojedavillarroel@yahoo.es, andres.mansilla@umag.cl

2 Instituto de Ecología y Biodiversidad (IEB), Santiago, Chile 
average of the macroalgae, per quadrant, in Santa Ana have a significant increase during summer, with the greatest richness in autumn $(p<0.05)$, meanwhile, Santa María did not show significant differences between the different seasons ( $p>0.05)$. The multivariate analysis showed significant differences in the assemblage composition of macroalgae between the two localities at each season $(p<0.05)$. This work is the first report of the seasonal dynamics of assemblages of subtidal macroalgae in the Strait of Magellan.

Key words: Subantarctic, macroalgae, biodiversity, seasonal, subtidal.

\section{INTRODUCCIÓN}

Los hábitats rocosos ofrecen varios desafíos, a la hora de evaluar el estado de las comunidades costeras. Dentro de las características más importantes de estos hábitats, se encuentra una alta variabilidad espacial y temporal de factores ambientales, que dan como resultado múltiples estructuras comunitarias, que pueden cambiar con el tiempo, o generar incluso parches dentro del mismo hábitat (Murray et al. 2016). Asimismo, las comunidades algales costeras están sujetas a procesos físicos (e.g. acción del oleaje, inestabilidad del substrato, desecación (Contreras-Porcia et al. 2011), temperatura, fotoperiodo, ph entre otros (Dawes, 1998) y biológicos (e.g. herbivoría), los cuales determinan la composición y abundancia, de las especies que alli habitan (Yodzis, 1993).

Dentro los factores que afectan la estructura de las comunidades algales, la estacionalidad es uno de los más relevantes (Cubit, 1984). En el caso de los hábitats intermareales se ha descrito que la variación estacional de las comunidades de macroalgas depende de la latitud y de factores ambientales locales (Hill \& Hawkins, 1991; Gunnarsson \& Ingolfsson, 1995; Thompson, 2000; Jenkins, 2001). Por ejemplo en zonas templadas del Hemisferio Norte, en las costas de Oregon, se ha descrito que durante el periodo invernal las macroalgas presentan un rápido crecimiento, mientras que durante los meses estivales, debido a la alta desecación y a la presión de herbivoría, estas comunidades reducen su cobertura (Cubit, 1984). Sin embargo, para las costas de California se ha descrito que la mínima cobertura de macroalgas se encuentra en invierno y la máxima en el verano tardío, estas variaciones han sido asociadas a periodos de surgencias costeras (Murray \& Horn, 1989). Este mismo patrón se ha observado hacia latitudes altas del Hemisferio Norte en las costas de Islandia, en donde la cobertura de macroalgas aumenta en verano y disminuye en invierno estos cambios se correlacionan con la irradianza y nutrientes, pero no con la temperatura (Gunnarsson \& Ingolfsson, 1995). Para el caso de los hábitats submareales, los cuales no están sujetos a los mismos factores que los intermareales (e.g. alta intensidad de luz y desecación), también se han descrito variaciones estacionales en la composición de las comunidades algales. Por ejemplo, en las costas de bahía La Paz, México, se ha observado que la mínima riqueza y abundancia de macroalgas se encuentra en invierno y la máxima en el verano tardío, debido a la alta variación de temperatura de esta zona (Cruz-Ayala et al. 1998). Patrón contrario ocurre en costas de la isla Jeju, Corea del Sur, donde la estacionalidad marca los patrones de riqueza de la flora submareal, presentando entre invierno y primavera la riqueza más alta, siguiéndole el verano temprano (Chan \& Sook, 2012). Para el caso de latitudes altas del Hemisferio Sur, en las costas de la península Antártica, donde las variaciones de luz y temperatura están estrictamente ligadas a la estacionalidad, las mayores coberturas de macroalgas se observan en periodos estivales (Kim, 2001), ya que a finales de primavera y comienzo del verano, las algas se encuentran en un estado óptimo fisiológico, debido a que las condiciones ambientales son ideales para un alto nivel de fotosíntesis (Becker et al. 2010). Por lo tanto, en general debido al efecto de la latitud y a condiciones ambientales locales, hacia altas latitudes en ambos hemisferios se ha descrito cambios estacionales notables en la riqueza y abundancia de comunidades de macroalgas costeras (Ojeda, 2013).

Para el sistema de canales y fiordos subantárticos $\left(43^{\circ} \mathrm{S}\right.$ a $\left.56^{\circ} \mathrm{S}\right)$, gran parte del conocimiento ficológico comenzó a gestarse en numerosas expediciones científicas realizadas desde el siglo XIX (ver Ramírez, 2010; Mansilla et al. 2013). No obstante, estudios sobre la 
caracterización de ensambles de macroalgas han sido escasos (Guzman \& Ríos, 1981; Ríos \& Mutschke, 1999; Benedetti-Cecchi \& Cinelli, 1997). Recientemente Ojeda (2013), realizó una caracterización temporal y espacial de la comunidad de macroalgas en Isla Navarino, encontrando que los ensambles de macroalgas no presentan un patrón de estructuración espacial, e incluso pueden existir diferencias en la estructura de los ensamble de macroalgas entre sitios, para similares niveles intermareales dentro de una misma bahía. Asi mismo, este autor encontró que los ensambles de macroalgas presentaron una marcada dinámica estacional, encontrándose la mayor riqueza y abundancia en verano, en relación a los periodos invernales. Sin embargo, todas estas contribuciones se han realizado en ambientes intermareales. El único trabajo que evalúa ensambles de macroalgas submareales es el realizado por Mansilla et al. (2013), el cual registró que la dinámica de los parámetros físicos presentes en las diferentes localidades dentro de la provincia Magallánica, influye de manera directa en la composición de la flora marina bentónica. No obstante, este trabajo evaluó comparaciones espaciales pero no temporales.

A pesar del conocimiento que existe sobre los ensambles de macroalgas, en su mayoría se han centrado en ambientes intermareales, en la caracterización del taxón y no en la búsqueda de asociaciones comunitarias. Algunos aportes sobre asociaciones comunitarias entre macroalgas y fauna, han sido los referidos para praderas de Macrocystis pyrifera (Ojeda \& Santelices, 1984; Adami \& Gordillo, 1999; Ríos et al. 2007; Mansilla et al. 2009; Rosenfeld et al. 2014). Recientemente se ha reportado que praderas naturales de Gigartina skottsbergii, presentes en la región de Magallanes albergan una gran diversidad de moluscos asociados a ella (Rosenfeld et al. 2015), enfatizando aún más en la importancia de las comunidades de macroalgas. Sin embargo, un punto relevante es que G. skottsbergii es la única macroalga en la región de Magallanes, que presenta una extracción comercial masiva. Esta especie se caracteriza por formar densas praderas sublitorales, con biomasas de alrededor de $1.773 \mathrm{~g} / \mathrm{m}^{2}$ y densidades de 15 individuos $/ \mathrm{m}^{2}$ por pradera (Ávila et al. 2004). La extracción de esta especie tiene por objeto proveer de materia prima a la industria procesadora de hidrocoloide carragenano (carragenina), gel de múltiples aplicaciones utilizado principalmente en la industria alimenticia y cosmética (Romo et al. 2001). En razón a la creciente demanda, tanto por parte de la industria nacional como por la necesidad de materia prima desde el extranjero, las praderas naturales han sufrido gran impacto y su recuperación ha sido lenta, mostrando indicios de la devastación de las praderas naturales en la región de Puerto Montt, Chile $\left(41^{\circ} \mathrm{S}\right)$. Por esta razón, en la actualidad buena parte de la presión extractiva se ha desplazado hacia el sur, en primera instancia hacia el área del Golfo de Penas $\left(\sim 47^{\circ} \mathrm{S}\right)$ y posteriormente hacia la Región de Magallanes ( $53^{\circ} \mathrm{S}$; Romo et al. 2001; Mansilla et al. 2008).

Actualmente hay escasa información sobre dinámicas temporales de ensambles submareales de macroalgas en la región de Magallanes y la constante extracción de poblaciones naturales de G. skottsbergii, constituye una excelente alternativa para estudiar la flora asociada, entregando así un registro de las especies asociadas y contribuyendo potencialmente a definir aspectos relacionados a la conservación (Gray, 1997; Fernández et al. 2000; Lancellotti \& Vásquez, 2000). En este sentido, el objetivo de este trabajo es describir la composición de las especies de macroalgas asociadas a dos praderas naturales de G. skottsbergii en el Estrecho de Magallanes, evaluando cambios temporales y espaciales de los ensambles.

\section{METODOLOGÍA}

\section{Descripción del área de estudio}

El área de estudio comprendió dos praderas naturales de Gigartina skottsbergii localizadas a ambos lados del Estrecho de Magallanes (Fig. 1): Santa María (S.M), ubicada en Tierra del

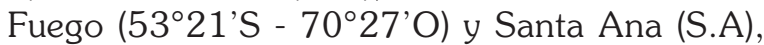
ubicada en la Península de Brunswick, Estrecho de Magallanes (Valdenegro \& Silva, 2003), zona que recibe influencias de aguas subantárticas desde el Pacífico, que van ingresando hacia el 


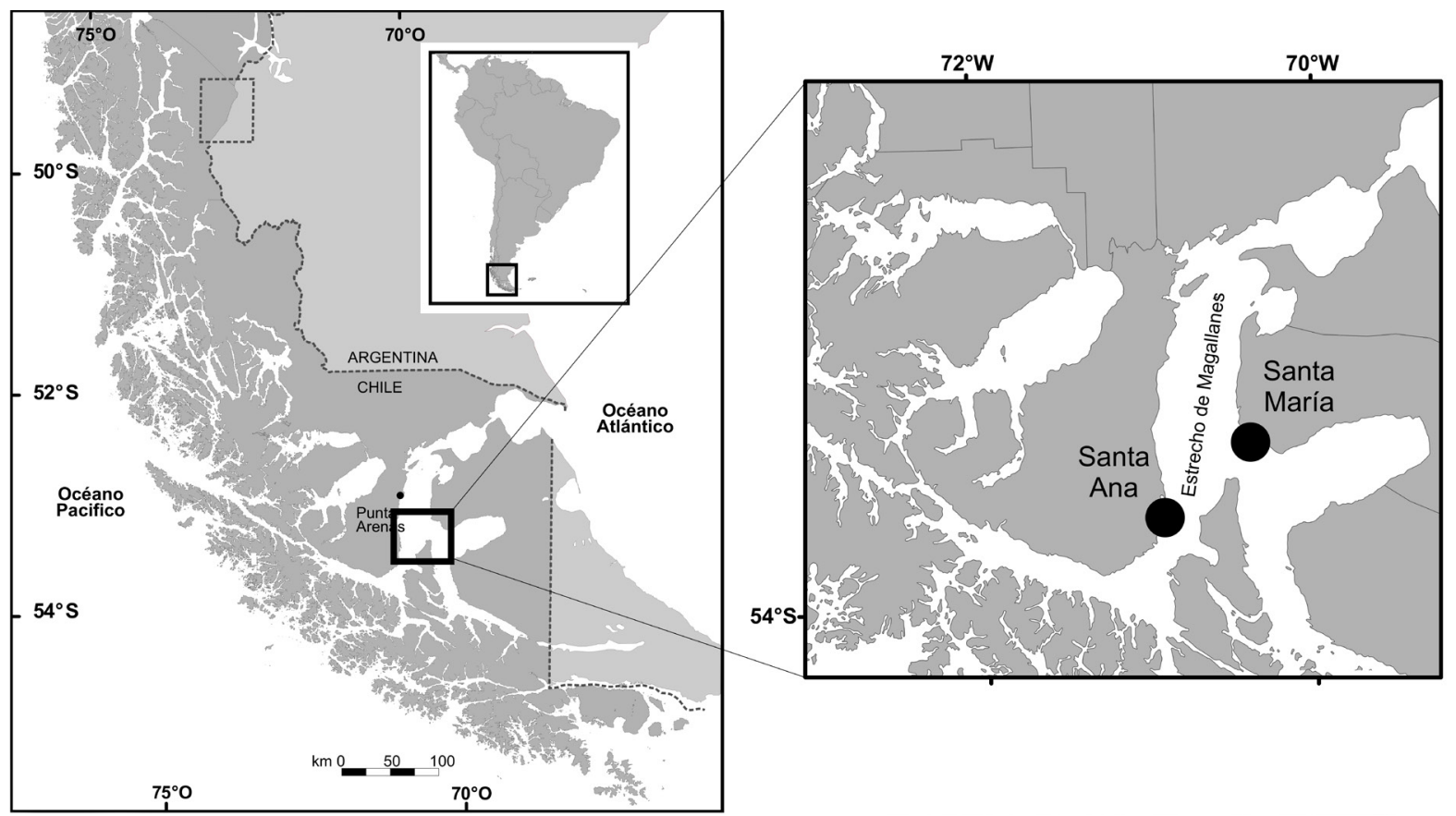

Fig. 1. Área de estudio. Localización de las praderas: Santa Ana y Santa María (en círculos).

interior del estrecho y se mezclan con aguas menos saladas, generando aguas subantárticas modificadas.

\section{Metodología de terreno}

Se realizó una georreferenciación mediante el sistema GPS Garmin 12, para cada sitio de estudio, con el objetivo de determinar el ancho y largo de las praderas de Gigartina skottsbergii.

Para el estudio de macroalgas asociadas a praderas de G. skottsbergii se realizaron muestreos extractivos en las praderas naturales de Punta Santa Ana y Santa María, de forma estacional (otoño, invierno, primavera, verano) durante un año, a fin de tener cuatro eventos de muestreos en total para cada pradera. Las muestras fueron extraídas mediante buceo autónomo, utilizando cuadrantes de $0,25 \mathrm{~m}^{2}$, los cuales fueron dispuestos al azar dentro de la pradera. En total se extrajeron 15 cuadrantes por muestreo. Posteriormente las muestras fueron depositadas en bolsas plásticas y preservadas en formalina diluida al 4-5\% en agua de mar, tamponada con borato de sodio.

Las muestras se analizaron en el laboratorio de Macroalgas Antárticas y Subantárticas (Lmas) Universidad de Magallanes. La identificación de muestras, se realizó mediante una caracterización y una descripción de caracteres morfológicos macroscópicos y microscópicos, utilizándose un microscopio estereoscópico marca Olympus modelo SZ61 y, un microscopio óptico marca Olympus, modelo CX31. Posterior a su identificación, se procedió a realizar excicatas de las muestras, utilizando la metodología indicada por Ramírez (1995). Para la identificación mediante bibliografía especializada, se utilizaron los aportes de Skottsberg (1941), Ramírez y Santelices (1991), Wiencke y Clayton (2002), Boraso de Zaixso (2004), Kim et al. (2004) y Guiry y Guiry (2013).

\section{Análisis de datos}

Para determinar la composición de macroalgas asociadas a praderas de $G$. skottsbergii de ambos sitios -y su comparaciónse realizaron análisis univariados y multivariados de biodiversidad utilizando el programa PRIMER 5.0. Dentro de los univariados se calculó para cada sitio: (a) la riqueza de especies (S) expresado simplemente como el número total de especies 
identificadas en $2.500 \mathrm{~cm}^{2}$. Para evidenciar diferencias significativas en la riqueza, promedio presentes en los dos sitios de muestro, tanto temporalmente como espacialmente, se realizó un PERMANOVA factorial de 2 vías. Para variables univariadas se calculó la distancia euclidiana entre pares de observaciones (Claudet et al. 2006). permutaciones sin restriccidatos.

La importancia específica de las distintas especies de macroalgas en cada localidad y en cada tiempo de muestreo se determinó mediante el análisis multivariado (SIMPER) (ver Clarke, 1993).

Para evidenciar diferencias significativas en la composición de los ensambles de macroalgas presentes en las praderas de G. skottsbergii, temporalmente como espacialmente y entre especies, se realizó un PERMANOVA factorial de 2 vías. Se calculó la disimilitud de Bray-Curtis entre pares de observaciones. Los datos fueron transformados a raíz cuarta y se usaron 9.999 permutaciones sin restricciones de datos.

Se utilizaron los modelos de acumulación de especies de Clench y de Dependencia Lineal como diagnóstico inicial (teórico) para determinar el esfuerzo de muestreo (Soberón \& Llorente, 1993). Todas las muestras fueron aleatorizadas para no afectar la forma de la curva (Colwell \& Coddington, 1994; Moreno \& Halffter, 2000). Para la estimación de los coeficientes de cada modelo de regresión no lineal, se utilizó el método de estimación Simplex y Quasi-Newton del paquete estadístico STATISTICA 7.

Como una manera complementaria se utilizaron también modelos no paramétricos de acumulación de especies, utilizando el programa ESTIMATE 9.0 y el índice de riqueza Chao 2 y de Jacknife 1.

\section{RESULTADOS}

El análisis taxonómico permitió la identificación de un total de 31 especies de macroalgas asociadas a ambas praderas naturales de G. skottsbergii; La pradera de Santa Ana, registró 28 especies, mientras que Santa María presentó un total de 26 especies de algas. (Tabla $1)$.

De la división Chlorophyta la especie con mayor frecuencia en ambas localidades, fue Ulva lactuca Linnaeus, perteneciente a la familia Ulvaceae, presente en todas las estaciones a excepción de Invierno. Las especies Codium dimorphum Svedelius y Ulva rigida C. Agardh, se encuentran solo en Santa Ana. Estas tres especies, se encuentran presentes durante otoño, habitando la misma localidad Santa Ana.

Macroalgas pertenecientes a la división Ochrophyta (Clase Phaeophyceae), se encuentran presentes en ambas localidades. Es la especie Ectocarpus siliculosus Lyngbye, la que presenta mayor ocurrencia encontrándose durante todo el año, en ambas localidades. Las demás especies de algas pardas ocurrieron en ambas localidades a excepción de la especie Desmarestia confervoides (Bory) M. E. Ramírez $\&$ A. F. Peters, la cual solo se presentó en Santa Ana (Tabla 1).

Las familias más frecuentes de la división Rhodophyta fueron Gigartinaceae (5 especies), Delesseriaceae (4 especies), Kallymeniaceae y Plocamiaceae cada una con 2 especies, las familias restantes solo presentaron una especie. La especie Iridaea cordata (Turner) Bory de Saint-Vincent, se encontró en ambas localidades, durante todas las estaciones del año (Tabla 1). Mientras que las especies Ptilonia magellanica (Montagne) J.Agardh, Callophyllis atrosanguinea (J. D. Hooker \& Harvey) Hariot y Plocamium cartilagineum (Linnaeus) P. S. Dixon se encontraron solo en Santa Ana, presentando variaciones estacionales en términos de ocurrencia. En general las macroalgas pertenecientes a la división Rhodophyta presentaron mayor ocurrencia en Santa Ana, registrándose en las estaciones otoño, primavera y verano, el mayor número de especies con 15 para cada estación, mientras que Santa María tiene el mayor número de especies (14 especies), durante las estaciones otoño e invierno y la menor riqueza durante primavera (9 especies) (Tabla 1).

En término de promedio de riqueza de especies, Santa Ana presenta el promedio más alto durante otoño $4,2 \pm 0,3$ y el menor durante invierno 2,6 $\pm 0,54$. Mientras que la localidad de Santa María registró el promedio de riqueza

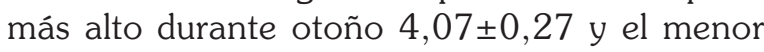
durante invierno 3,0 0,59 (Fig. 2b). 
Tabla 1. Ocurrencia estacional de macroalgas submareales asociadas a praderas naturales de G. skottsbergii, presentes en las localidades de Santa Ana (S.A) y Santa María (S.M), Magallanes, Chile. * indica presencia del taxón.

\begin{tabular}{lllllllll}
\hline & & Otoño & \multicolumn{2}{c}{ Invierno } & Primavera & \multicolumn{2}{c}{ Verano } \\
\hline Especie & S.A & S.M & S.A & S.M & S.A & S.M & S.A & S.M \\
\hline
\end{tabular}

División Chlorophyta

\section{Familia Codiaceae}

Codium dimorphum Svedelius

\section{Familia Ulvaceae}

Ulva rigida C.Agardh

Ulva lactuca Linnaeus

\section{División Ochrophyta}

Familia Desmarestiaceae

Desmarestia distans (C.Agardh) J.Agardh

Desmarestia confervoides (Bory) M.E.Ramírez \& A.F. Peters

\section{Familia Ectocarpaceae}

Ectocarpus siliculosus Lyngbye

\section{Familia Laminariaceae}

Macrocystis pyrifera (Linnaeus) C.Agardh

\section{Familia Lessoniaceae}

Lessonia flavicans Bory

\section{Familia Scytosiphonaceae}

Scytosiphon lomentaria (Lyngbye) Link

\section{Familia Stypocaulaceae}

Halopteris obovata (J.D.Hooker \& Harvey) Sauvageau

\section{División Rhodophyta}

\section{Familia Balliaceae}

Ballia callitricha (C.Agardh) Kützing

\section{Familia Bangiaceae}

Porphyra/Pyropia sp.

\section{Familia Bonnemaisoniaceae}

Ptilonia magellanica (Montagne) J.Agardh

\section{Familia Callithamnieae}

Callithamnion sp.

\section{Familia Ceramiaceae}




\begin{tabular}{llllllllll}
\hline & \multicolumn{4}{c}{ Otoño } & \multicolumn{2}{c}{ Invierno } & \multicolumn{2}{c}{ Primavera } & \multicolumn{2}{c}{ Verano } \\
\hline Especie & S.A & S.M & S.A & S.M & S.A & S.M & S.A & S.M \\
\hline
\end{tabular}

Familia Dasyacea

Heterosiphonia sp.

\section{Familia Delesseriaceae}

Hymenena sp.

Phycodrys sp.

Myriogramme livida (J.D.Hooker \& Harvey) Kylin

Cladodonta lyallii (J.D.Hooker \& Harvey) Skottsberg

\section{Familia Gigartinacea}

Iridaea cordata (Turner) Bory de Saint-Vincent

Rhodymenia sp.

Rhodymenia coccocarpa (Montagne) M.J.Wynne

Mazzaella laminarioides (Bory de Saint-Vincent) Fredericq

Sarcothalia crispata (Bory de Saint-Vincent) Leister

\section{Familia Halymenia}

Grateloupia sp.

\section{Familia Kallymeniaceae}

Callophyllis variegata (Bory de Saint-Vincent) Kützing

Callophyllis atrosanguinea (J.D.Hooker \& Harvey) Hariot

\section{Familia Plocamiaceae}

Plocamium cartilagineum (Linnaeus) P.S.Dixon

Plocamium sp.

\section{Familia Rhodomelaceae}

La variación estacional de la estructura del ensamble de macroalgas en cuanto a la riqueza de especies promedio presentó cambios significativos estacionales (Fig. 2), principalmente entre verano $e$ invierno y entre otoño e invierno en la localidad de Santa Ana (ver tabla 2) (Permanova: $\mathrm{F}=20,6156 ; \mathrm{P}=0,001$ ), ya que en la localidad de Santa María aunque se observó una disminución en la riqueza promedio de especies en el periodo invernal, estas diferencias no fueron significativas (Tabla 2). Espacialmente no se observó diferencias significativas entre las dos praderas evaluadas, para cada una de las estaciones muestreadas en el periodo de estudio (Tabla 2).

La importancia específica de cada especie por estación, determinado por el porcentaje de contribución SIMPER, no mostró el mismo patrón en ambas localidades, ya que, en Santa María se observaron mayores cambios en la identidad y riqueza de los taxones que más contribuyeron al ensamble estacional en términos de composición de especies. Estas variaciones se observaron entre la estación de invierno frente al resto de las estaciones. Por ejemplo, durante el invierno solamente 3 taxones, totalizaron el $70 \%$ de contribución, siendo Phycodrys sp. y Callophyllis variegata las 


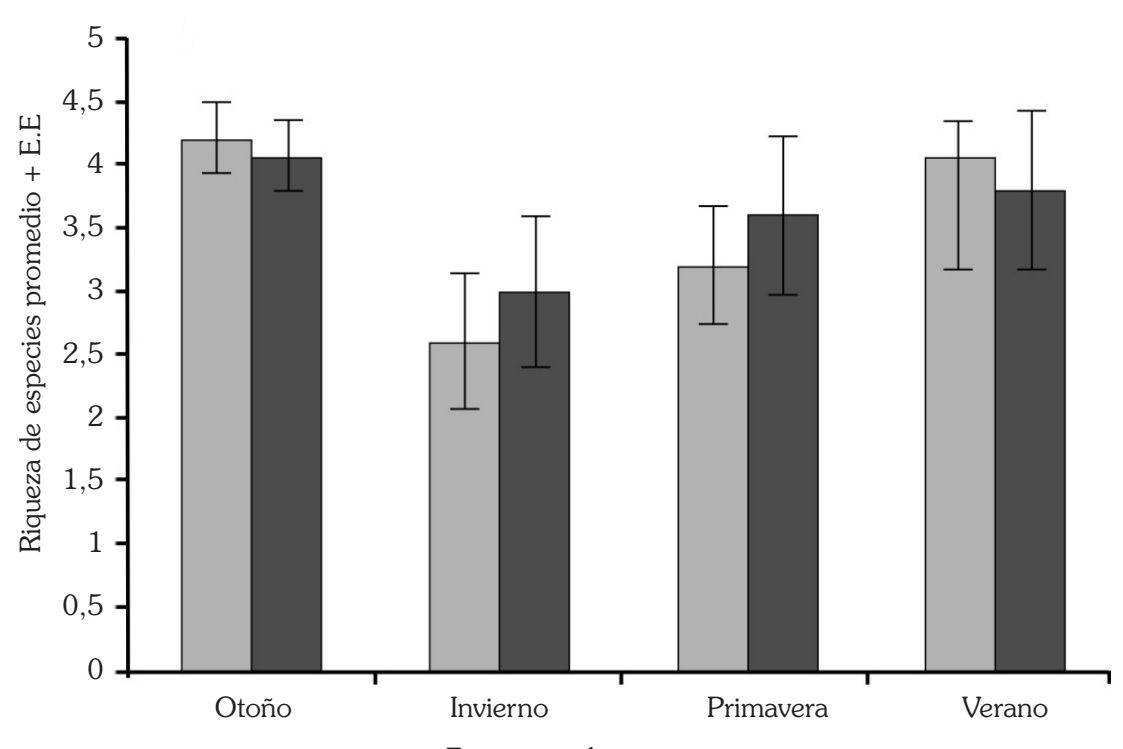

Estaciones de muestreo

Punta Santa María

Punta Santa Ana

Fig. 2. Riqueza promedio estacional en las localidades de Santa Ana, y Santa María, Magallanes, Chile.

Tabla 2. Análisis de permutaciones (PERMANOVA) para la riqueza promedio de los ensambles de macroalgas asociadas a G. skottsbergii. El diseño fue de tipo factorial considerando localidad y estación. Valores de $P$ para las comparaciones post-hoc entre las estaciones para cada localidad. Los datos se basaron en distancia Euclidiana y se realizaron 9999 permutaciones. Números en negrita indican diferencias significativas $(p<0,05)$.

\begin{tabular}{ccccc}
\hline Fuente & $\mathrm{df}$ & $\mathrm{MS}$ & $\mathrm{F}$ & $P$ \\
\hline Localidad & 1 & 0,3 & 0,00857 & 0,7505 \\
Estación & 3 & 10,711 & 3,0582 & $\mathbf{0 , 0 3 0 5}$ \\
Lo x Ti & 3 & 0,92222 & 0,26331 & 0,8535 \\
Res & 112 & 3,5024 & & \\
\hline Total & 119 & \multicolumn{2}{c}{ Localidad } \\
\hline Estaciones & \multicolumn{2}{c}{ Localidad } \\
\hline \multicolumn{2}{c}{ S.A } \\
\hline \multicolumn{2}{c}{$\mathrm{t}$} & \multicolumn{2}{c}{$\mathrm{T}$} & \multicolumn{1}{c}{ S } \\
\hline Otoño, Invierno & 2,5923 & $\mathbf{0 , 0 1 8 5}$ & 1,6391 & 0,1326 \\
Otoño, Primavera & 1,8286 & 0,1028 & 0,69554 & 0,5429 \\
Otoño, Verano & 0,33466 & 0,8717 & 0,38773 & 0,7772 \\
Invierno, Primavera & 0,84452 & 0,4647 & 0,70156 & 0,5331 \\
Invierno, Verano & 2,4295 & $\mathbf{0 , 0 3 2 6}$ & 0,92113 & 0,3955 \\
Primavera, Verano & 1,6305 & 0,1452 & 0,22632 & 0,8887 \\
\hline
\end{tabular}



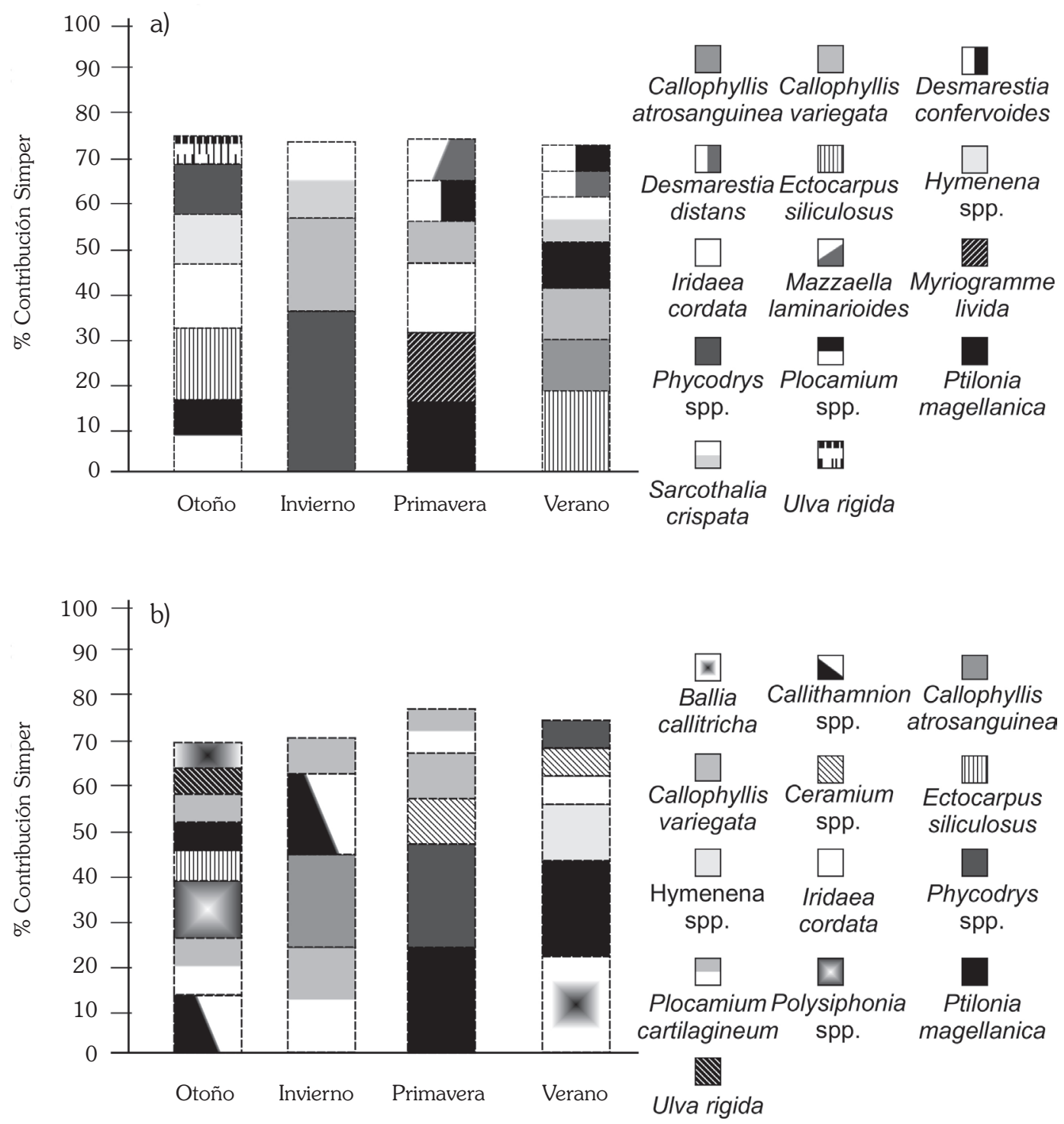

Fig 3. Porcentaje de contribución SIMPER de macroalgas estacionales para las dos localidades, a) corresponde Santa María y b) a Santa Ana. El límite de contribución fue de un $70 \%$ del total del ensamble de macroalgas.

macroalgas responsables de este mayor porcentaje (Fig. 3a). Mientras que durante otoño, primavera y verano, entre 6 y siete taxones respectivamente, conformaron el $70 \%$ de contribución, siendo Plocamium sp. el alga de mayor porcentaje en otoño, Ptilonia magellanica en primavera, y Ectocarpus siliculosus en verano (Fig. 3b). Para el caso de la localidad de Santa Ana, no se observaron mayores cambios en la identidad de los taxones presentes en las 4 estaciones (Fig. 3b). Por ejemplo, durante todas las estaciones entre 4 y 8 taxones, totalizaron el $70 \%$ de contribución, siendo Ptilonia magellanica, Plocamium cartilagineum, y Callophyllis variegata macroalgas presentes en casi todas las estaciones de muestreo y responsables de la contribución a sus respectivos grupos (Fig. 3b).

El análisis multivariados Permanova arrojo diferencias significativas temporales y espaciales en la composición de los ensambles de macroalgas 
Tabla 3. Análisis de permutaciones (PERMANOVA) para diferentes variables de los ensambles de macroalgas asociadas a G. skottsbergii. El diseño fue de tipo factorial considerando localidad y estación. Valores de $P$ para las comparaciones post-hoc entre las estaciones para cada localidad y entre localidades para cada estación. Los datos se basaron en la disimilitud de BrayCurtis y se realizaron 9999 permutaciones. Números en negrita indican diferencias significativas $(p<0,05)$.

\begin{tabular}{|c|c|c|c|c|}
\hline Fuente & $\mathrm{df}$ & MS & $\mathrm{F}$ & $P$ \\
\hline Localidad & 1 & 22454 & 6,7421 & 0,0001 \\
\hline Estación & 3 & 5585,3 & 1,6771 & 0,0134 \\
\hline Lo $\times \mathrm{Ti}$ & 3 & 10494 & 3,151 & 0,0001 \\
\hline Res & 97 & 3330,4 & & \\
\hline Total & 104 & & & \\
\hline Fuente & \multicolumn{2}{|c|}{ Localidad } & \multicolumn{2}{|c|}{ Localidad } \\
\hline \multirow[t]{2}{*}{ Estaciones } & \multicolumn{2}{|c|}{ P.S.A } & \multicolumn{2}{|c|}{ P.S.M } \\
\hline & $\mathrm{t}$ & $P$ & $\mathrm{t}$ & $P$ \\
\hline Otoño, Invierno & 0,86866 & 0,6323 & 1,9087 & 0,0087 \\
\hline Otoño, Primavera & 1,1617 & 0,2427 & 1,6906 & 0,0085 \\
\hline Otoño, Verano & 1,2927 & 0,1589 & 1,3906 & 0,0661 \\
\hline Invierno, Primavera & 1,3292 & 0,1384 & 3,0085 & 0,0072 \\
\hline Invierno, Verano & 1,3004 & 0,1173 & 2,2849 & 0,0099 \\
\hline Primavera, Verano & 1,623 & 0,5095 & 1,9753 & 0,0179 \\
\hline
\end{tabular}

\begin{tabular}{ccc}
\hline Fuente & \multicolumn{2}{c}{ Localidades } \\
\hline \multicolumn{2}{c}{ P.S.A x P.S.M } \\
\hline Estaciones & $\mathrm{t}$ & $P$ \\
\hline Otoño & 1,4225 & $\mathbf{0 , 0 1 6 2}$ \\
Invierno & 2,1769 & $\mathbf{0 , 0 0 9 6}$ \\
Primavera & 2,8381 & $\mathbf{0 , 0 0 8 1}$ \\
Verano & 2,4969 & $\mathbf{0 , 0 0 6 6}$
\end{tabular}

(Tabla 3). No obstante, en la localidad de Santa Ana no se observaron diferencias significativas a lo largo del año (Tabla 3). Distinto es el caso de la localidad de Santa María, en donde se evidenciaron diferencias significativas entre todas las estaciones, excepto entre verano y otoño (Tabla 3). En relación a las comparaciones espaciales entre localidades, se observaron diferencias significativas en todas las estaciones del año (Tabla 3).

La predicción estimada para la riqueza de especies asociadas al esfuerzo de muestreo para la localidad de Santa Ana determinada mediante el modelo de Clench, mostró que los valores de las constantes fueron $\mathrm{a}=5,543288 \mathrm{y} \mathrm{b}=0,168482$. La relación de estos valores (a/b) arrojó una riqueza máxima esperable de 32,9 especies (valor de la asíntota de la curva de acumulación de especies con un R2 $=0,98$ ), valor superior en 4 especies al observado en este estudio. En cuanto, al modelo de dependencia lineal, las constantes fueron $\mathrm{a}=$ 3,559790 y b $=0,123327$; por lo tanto la riqueza máxima esperable $(\mathrm{a} / \mathrm{b})$, fue de 28,9 especies con un $\mathrm{R} 2=0,98$, obteniendo el mismo valor al observado empíricamente en el presente estudio (Fig 4a). Por lo tanto, este es el modelo teórico que predijo exactamente el número observado de especies de macroalgas en forma empírica, para esta localidad. Para el caso de la localidad de Santa María, el modelo de Clench, mostró que los valores de las constantes fueron $\mathrm{a}=2,608430$ y $\mathrm{b}=$ 
0,086784. La relación de estos valores (a/b) arrojó una riqueza máxima esperable de 30,2 especies (valor de la asíntota de la curva de acumulación de especies con un $\mathrm{R} 2=0,98$ ), valor superior en 4 especies al observado en este estudio. En cuanto, al modelo de dependencia lineal, las constantes fueron $\mathrm{a}=1,937550$ y $\mathrm{b}=0,078706$; por lo tanto la riqueza máxima esperable $(\mathrm{a} / \mathrm{b})$, fue de 24,6 especies con un R2 $=0,98$, obteniendo un valor inferior al observado empíricamente en el

a)

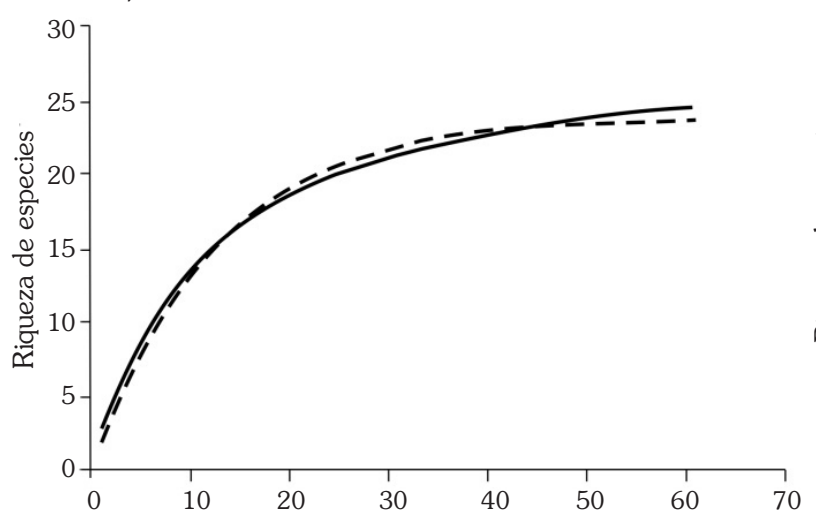

Modelo de Clench

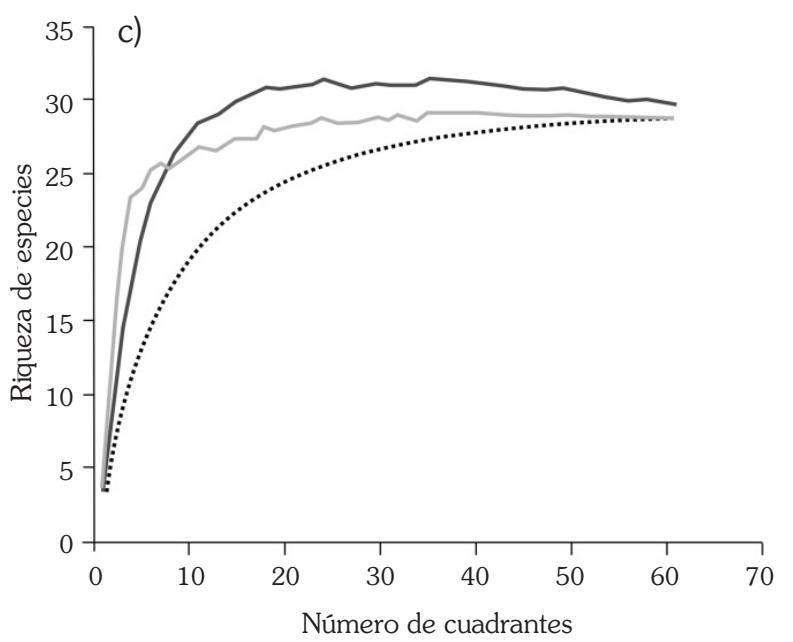

presente estudio (Fig 4b).

Para el caso de los modelos no paramétricos se observó que, para el caso de la localidad de Santa Ana el Chao 2 estimo un valor de 29,125, mismo valor que el observado en el campo. Asimismo, se puede observar como la curva de Chao 2 se junta con la de S obs (Fig 4c). Sin embargo, el modelo de Jacknife 1 estimó un total de 29,98, valor superior en una especie al observado en el campo, y a diferencia del Chao 2, la curva de Jacknife 1 quedo
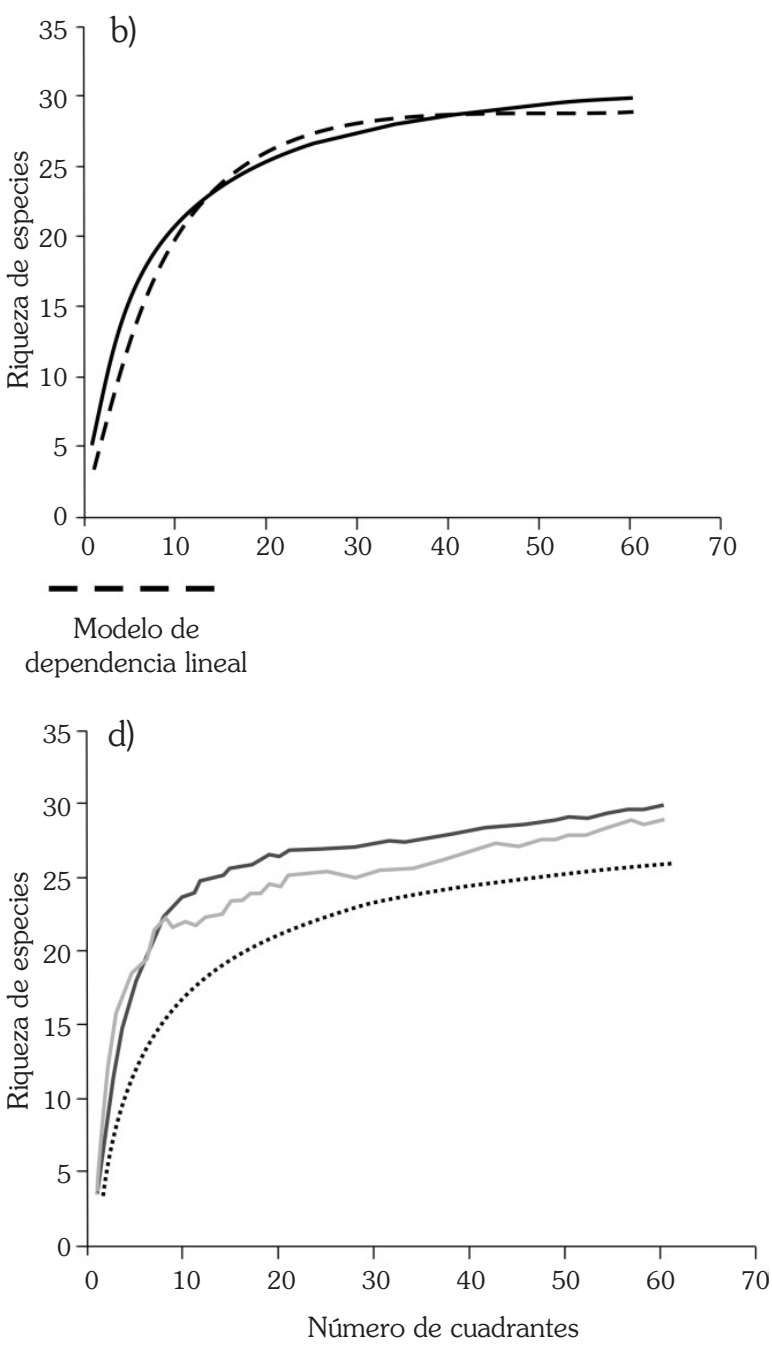

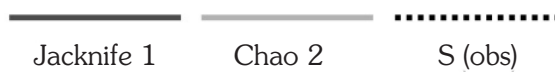

Fig 4. a-c) Curvas de acumulación de especies de macroalgas según los estimadores paramétricos, Clench y dependencia lineal y según los estimadores no paramétricos Chao 2 y Jack 1 para la localidad de Santa Ana, b-d) curvas de acumulación de especies de macroalgas según los estimadores paramétricos, Clench y dependencia lineal y según los estimadores no paramétricos Chao 2 y Jack 1 para la localidad de Santa María. 
por sobre la de S obs (Fig. 4c). En la localidad de Santa María, ambos modelos estimaron una riqueza esperada superior a la observada en el campo (Chao 2 = 28,25; Jacknife $1=29,96$ ), y ambas curvas quedaron por sobre la del S obs (ver Fig. 4d).

\section{DISCUSIÓN}

Esta línea de base sobre macroalgas asociadas a Gigartina skottsbergii, ha fomentado la necesidad, y urgencia de la gestión a entornos costeros, ya que a pesar de la importancia económica de este recurso natural, estas praderas presentan una alta diversidad de especies asociadas, ocupando un papel ecológico importante en los ecosistemas costeros. La riqueza arrojada por el modelo de dependencia lineal para las dos localidades fue distinta, ya que, para Santa Ana la riqueza esperada fue la misma que la observada en terreno (28 especies), mientras que en Santa María la riqueza esperada fue menor. Por otro lado el modelo de Clench para ambos sitios arrojó el mismo resultado, la riqueza esperada fue mayor que la observada en terreno. Esta situación se podría explicar debido a que aún falta mayor conocimiento sobre la taxonomía de muchos grupos de macroalgas, muchas especies quedan indeterminadas y no son incluidas en los listados o no son reconocidas en el terreno, y según Soberón y Llorente (1993) en el modelo de Clench la probabilidad de encontrar una nueva especie aumentará según la experiencia en el terreno. Por lo tanto, el modelo de Clench sugiere aumentar el esfuerzo de muestreo pero a una escala espacial y temporal más amplia para alcanzar la asíntota en la estimación de las especies de macroalgas sublitorales del Estrecho de Magallanes.

Para el caso de los modelos no paramétricos, también se encontraron resultados contrastantes, debido a que, en Santa Ana el Chao 2 estimo el mismo valor de riqueza que el observado en terreno (28 especies), mientras que en Santa María este modelo estimo un valor superior al observado en terreno. Asimismo, el modelo de Jacknife 1 en ambas localidades estimo valores de riqueza superiores al observado en terreno. Estos modelos no paramétricos trabajan en base al número de Uniques (número de especies que ocurren solamente en una muestra) y Duplicates (número de especies que ocurren en exactamente dos muestras). Esto se basa en el supuesto de que en los ecosistemas los individuos de una especie no habitan solos, sino en poblaciones (Magurran, 1988), por lo tanto, si uno encuentra muchos unique en un muestreo, podría estar indicando que no se ha invertido un número suficiente de unidades muestreales. Por ende, en el caso particular de Santa María los modelos en general están sugiriendo invertir un mayor esfuerzo muestreal.

No obstante a lo anterior, es importante considerar que para evaluar el comportamiento de los distintos estimadores es necesario conocer el número de especies de la comunidad (Walther \& Moore, 2005; Gonzalez-Oreja et al. 2010). A menos que la comunidad se haya muestreado de modo exhaustivo, estas curvas podrían no trabajar adecuadamente (Magurran, 2004). Por ende, algunos autores recomiendan no trabajar solamente con un estimador, sino, probar varios modelos para ver cómo estos se comportan a los datos (Gonzalez-Oreja et al. 2010), ya que estos pueden variar, según las situaciones o para un grupo concreto de organismos (Walther \& Moore, 2005). En general, tras evaluar los resultados de los 4 modelos utilizados en este estudio, se puede inferir, que algunos se ajustaron bien en la localidad de Santa Ana (dependencia lineal y Chao 2), pero para Santa María en general la mayoría sugiere un mayor esfuerzo muestreal.

\section{Riqueza de especies}

Estudios previos han abordado la flora marina bentónica, en la región de Magallanes, estos estudios han registrado especies que habitan la tanto la zona del intermareal, y el submareal; Ramírez (2010) registra para ambas zonas 234 especies de macroalgas para la región de Magallanes, y Tierra del Fuego, Ojeda (2013) registra 49 especies presentes en la zona del intermareal de Bahía Róbalo, Isla Navarino, mientras que Mansilla et al. (2013) registra 57 especies en diferentes ambientes submareales. Este estudio registra un total de 31 especies, asociadas a praderas submareales naturales de Gigartina skottsbergii, correspondiendo a un 13,24 \% de las algas registradas por Ramírez (2010). 


\section{Composición y riqueza estacional}

Durante el ciclo anual, el $68 \%$ de las macroalgas que se encuentran asociadas a las dos praderas naturales de Gigartina skottsbergii, pertenecen a la división Rhodophyta. Estas especies presentan una alta tolerancia a la inmersión (Grime, 1977), por lo que son comunes en ambientes submareales, dominando así la franja sublittoral, en conjunto de algas pardas, como kelps (Lewis, 1964). Especies como Ectocarpus siliculosus, e Iridaea cordata, ocurrieron estacionalmente, en ambas praderas, se ha descrito que esta última en la región Antártica, es capaz de presentar variaciones en la eficiencia fotosintética anual, considerándose una ventaja adaptativa en torno a la estacionalidad, y a la zonificación vertical (Weykam et al. 1997; Lüder et al. 2001, 2003).

En términos de composición florística las algas asociadas a ambas praderas, presentan similar tendencia, disminuyendo la riqueza promedio hacia invierno. Esta variabilidad, puede explicarse debido a las condiciones de luz extremadamente bajas que ocurren durante el invierno en latitudes altas, las cuales afectan directamente a la zona submareal (Svendsen et al. 2002), en la región subantártica esto ha sido observado para la zona intermareal (Ojeda et al. 2013). La flora asociada en la pradera Santa Ana presentó cambios en los ensambles estacionales, entre otoño-invierno, y verano-invierno, mientras que la pradera de Santa María aunque muestra fluctuaciones en la riqueza promedio no es significativa.

Las diferencias entre ambas praderas, pueden estar influenciadas por un factor abiótico importante, la penetración del UV-B, en aguas claras durante la época invernal, y el daño biológico que este genera, principalmente este fenómeno ocurre en latitudes altas debido al derretimiento de hielos (Bischof et al. 1998), y en la región subantártica puede ocurrir debido a la disminución en la cobertura de bosques de Macrocystis pyrifera, alga a la cual se encuentra asociada la pradera de G. skottsbergii de Santa Ana. Variaciones de luz, y UV, que no presenta la pradera de Santa María, ya que no se encuentra asociada a kelps.

La flora asociada a la pradera Santa María, presentó variaciones en la identidad de los taxones, siendo las algas responsables de estos cambios Phycodrys sp. y Callophyllis variegata durante invierno, Plocamium sp. otoño, Ptilonia magellanica en primavera y Ectocarpus siliculosus en verano. En relación a la presencia de estas especies, está dado debido a sus ciclos de vida, por ejemplo Phycodrys sp., y C. variegata, la cual presenta un ciclo polysifónico trifásico estacional presentando un aumento de los estadíos reproductivos entre junio a marzo, y posteriormente disminuyendo (Mansilla et al. 2012), mientras que especies como E. siliculosus, presentan mayor presencia durante los meses de primavera tardía-verano (Soto, 1991). No se observó cambios en el ensamble estacional de Santa Ana, siendo las algas más representativas Ptilonia magellanica, Plocamium cartilagineum, y Callophyllis variegata, las cuales contribuyeron, a que el ensamble no modifique su composición. P. magellanica es característica de substratos rocosos, como el que se presenta en Santa Ana (Mendoza \& Nizovoy, 2000; Soto et al. 2012).

\section{Variaciones espaciales}

En términos de cambio espacial, se presentaron variaciones entre Santa AnaSanta María: durante otoño se registraron especies como Callithamnion sp., Plocamium cartilagineum, Callophyllis atrosanguinea, y Ptilonia Magellanica, especies que no se encontraron en el ensamble de Santa María, en invierno las especies que aportaron a la disimilitud entre praderas fueron Phycodrys sp., y Sarcothalia crispata, durante primavera las especies fueron Myriogramme libida, e Iridaea cordata, mientras que en verano la especie que aportó en la disimilitud fue Desmarestia confervoides, registrándose solo en Santa Ana. Cabe destacar que la variación espacial que presentaron ambos ensambles de algas, se encuentran sujetos a los ciclos de vida, y factores internos de estas especies, como a variaciones en los factores abióticos de ambos sectores, los cuales influyen en la composición, y distribución de las algas bentónicas. Un ejemplo es la heterogeneidad del substrato, Santa María se caracteriza por presentar un substrato de rodolitos, mientras que Santa Ana presenta un substrato de terrazas, en consecuencia algunos 
estudios han observado que ocurre un aumento de riqueza de macroalgas en superficies duras, y estables, aptas para su colonización, mientras que esta riqueza tiende a disminuir, o a estar influenciada por un substrato más suave, blando o inestable (Klöser et al. 1996; Underwood, 2004; Wulff et al. 2009), como es el caso de los rodolitos. Un factor biótico importante es la presencia del kelp (Kirikki, 1996), como $M$. pyrifera, en Santa Ana que forma un canopy, generando protección a otras especies de macroalgas, además de variaciones en la herbivora (Rilov \& Schiel, 2011), y reclutamiento de algas (Reaugh-Flower et al. 2011), el efecto de estas últimas variaciones, aún no han sido evaluadas en ambas praderas. Mientras que a macroescala, el fotoperiodo, la temperatura y la pluviosidad (Cruz-Motta et al. 2010) pueden ser factores que afectan la riqueza espacial (Chappuis et al. 2014). En la Ecorregion subantártica de Magallanes se ha podido observar mediante estudios de macroalgas, que la dinámica de los parámetros físicos presentes en cada zona de muestreo, influye de manera directa en la composición de la flora marina bentónica (Mansilla et al. 2013) de cada localidad.

La alta tasa extractiva de G. skottsbergii, en la región de Magallanes, Chile, con fines comerciales (extracción de carragenina), ha llevado a la explotación de las praderas naturales, por lo cual no se conoce que especies se asocian a esta macroalga. El estudio realizado por Rosenfeld et al. (2015), en estas praderas naturales, arrojó un total de 42 especies de moluscos, presentando un alto índice de riqueza, siendo este el primer estudio de fauna asociada a praderas naturales de G. skottsbergii. Finalmente es importante destacar que este estudio contribuye de manera significativa al conocimiento local porque: a) entrega la primera información sobre flora asociada a praderas naturales de Gigartina skottsbergii, y b) entrega el primer inventario estacional de ensambles sublitorales de macroalgas en dos localidades de la micro-cuenca central de Estrecho de Magallanes, contribuyendo a un mejor conocimiento local sobre las dinámicas estacionales de ensambles de macroalgas sublitorales, como también promover la importancia de las praderas naturales de G. skottsbergii como sitios de alta diversidad de flora y fauna.

\section{AGRADECIMIENTOS}

Los autores agradecen a FONDEF CONICYT, Chile (Proyecto AQ08I1011). A MSc. Marcela Ávila, Universidad Arturo Prat, Puerto Montt, Chile, por su apoyo en el desarrollo de este trabajo. A María Eliana Ramirez, por su apoyo en el desarrollo de este trabajo. A las Facilidades y el equipamiento que otorgó el laboratorio de Macroalgas Antárticas, y Subantárticas (LMAS). J. Marambio, agradece a la beca entregada por el Instituto de Ecología y Biodiversidad (IEB) código PFB-23-2008. A. Mansilla agradece a la Iniciativa Científica Millennium ( $n^{\circ}$. P05-002 ICM, Chile), y al Programa de Financiamiento Basal, de la Comisión Nacional de Investigación Científica y Tecnológica ( $\mathrm{n}^{\circ} \mathrm{PFB}-23$, Chile).

\section{LITERATURA CITADA}

Adami, M. L., \& Gordillo, S. (1999). Structure and dynamics of the biota associated with Macrocystis pyrifera (Phaeophyta) from the Beagle Channel, Tierra del Fuego. Scientia Marina, 63(1), 183-191.

Anderson, M. J. (2001). A new method for nonparametric multivariate analysis of variance. Austral Ecology, 26, 32-46.

Ávila, A., Cáceres, J., Nuñez, M., Camus, P., Pavez, H., Cortes, H., González, J.,... Candia, A. (2004). Investigación y manejo de praderas de luga roja en la XII Región. Informe final proyecto FIP-IFOP 2002-27.

Becker, S., Graeve, M., \& Bishof K. (2010). Photosynthesis and lipid composition of the Antarctic endemic rhodophyte Palmaria decipiens, effects of changing light, and temperature levels. Polar Biology, 33, 945955.

Benedetti-Cecchi, L., \& Cinelli, F. (1997). Spatial distribution of algae and invertebrates in the rocky intertidal zone of the Strait of Magellan, are patterns general? Polar Biology, 18, 337-343.

Bischof, K., Hanelt, H., \& Wiencke C. (2009). UV-Radiation can affect depth-zonation of 
Antartic macroalgae. Marine Biology, 131, 597-605.

Boraso de Zaixso. (2004). Chlorophyta marinas de la argentina. Historia natural, 3(2), 95119.

Chan J., \& Sook, G. (2012). Seasonal variation in depth-stratified macroalgal assemblage patterns on Marado, Jeju Island, Korea. Algae, 2012, 27(4), 269-281.

Chappuis, E., Terradas, M., Cefalì M. E., Mariani, S., \& Ballesteros, C. (2014). Vertical zonation is the main distribution pattern of littoral assemblages on rocky shores at a regional scale. Estuarine Coastal and Shelf Science, 147, 113-122.

Clarke, K. R. (1993). Non-parametric multivariate analyses of changes in community structure. Australian Journal of Ecology, 18, 117143.

Claudet, J., Pelletier, D., Jouvenel, J. Y., Bachet, F., \& Galzin, R. (2006). Assessing the effects of marine protected area (mpa) on a reef fish assemblage in a Northwestern Mediterranean marine reserve: identifying community-based indicators. Biological Conservations, 130, 349-369.

Colwell, R. K., Mao, C. X., \& Chang, J. (2004). Interpolating, extrapolating, and comparing incidence-based species accumulation curves. Ecology, 85, 2717-2727.

Colwell, R. K., \& Coddington J. A. (1994). Estimating terrestrial biodiversity through extrapolation. Philosophical Transactions of the Royal Society of London Series, 345, 101-118.

Contreras-Porcia, L., Thomas, D., Flores V., \& Correa, J. A. (2011). Tolerance to oxidative stress induced by desiccation in Porphyra columbina (Bangiales, Rhodophyta). Journal experimental Botany, 62, 18151829

Cruz-Ayala, M. A., Casas-Valdez, M., \& OrtegaGarcia S. (1994). Temporal and Spatial Variation of Frondose Benthic Seaweeds in La Paz Bay, B. C. S., Mexico. Botánica Marina, 41, 191-198.

Cruz-Motta, J. J., Miloslavich, P., Palomo, G., Iken, K., Konar, B., Pohle, G., Trott, T.,... Shirayama, Y. (2010). Patterns of spatial variation of assemblages associated with intertidal rocky shores: a global perspective. PLoS ONE, 5, e14354.

Cubit, J. D. (1984). Herbivory and the seasonal abundance of algae on a high intertidal rocky shore. Ecology, 65, 1904-1917.

Dawes, C. J. (1998). Marine Botany. 2nd ed. John Wiley \& Sons, New York.

Fernández, M., Jaramillo, E., Marquet, P. A., Moreno, C. A., Navarrete, S. A., Ojeda, F. P., Valdovinos C., \& Vásquez, J. A. (2000) diversity, dynamics and biogeography of chilean benthic nearshore ecosystems: an overview and guidelines for conservation. Revista Chilena de Historia Natural, 73, 797-830.

González-Oreja, J. A, De la Fuente, A., Hernández, L., Buzo, D., \& Bonache, C. (2010). Evaluación de estimadores no paramétricos de la riqueza de especies. Un ejemplo con aves $\mathrm{n}$ áreas verdes de la ciudad de puebla, México. Animal Biodiversity and Conservation, 33, 31-45.

Gray, J. S. (1997). Marine biodiversity: patterns, threats and conservation needs. Biodiversity and Conservation, 6, 153-175.

Grime, J. P. (1977). Evidence for the existence of three primary strategies in plants and its relevance to ecological and evolutionary theory. American Naturalist. 111, 11691194.

Guiry, M. D., \& Guiry, G. M. (2013). Algaebase. World-wide electronic publication, national university of Ireland, Galway. [en línea] http://www.algaebase.org.

Gunnarsson, K., \& Ingólfsonn, A. (1995). Seasonal changes in the abundance of intertidal algae in southwestern Iceland. Botánica Marina, 38, 69-77.

Guzmán, L., \& Ríos, C. (1981). Estructura de macroorganismos de una playa de bloques y cantos de isla Wollaston, archipiélago del Cabo de Hornos. Anales del Instituto de la Patagonia, 12, 257-271.

Hill, A. S., \& Hawkin, J. (1991). Seasonal and spatial variation of epilithic micro algal distribution and abundance and its ingestion by Patella vulgata on a moderately exposed rocky shore. Journal Marine Biology, 71, 
403-423.

Jenkins, S. R., Arenas, F., Arrontes, J., Bussell, J., Castro, J., Coleman, R. A., Hawkins, S. J.,...Hartnoll R. G. (2001). Europeanscale analysis of seasonal variability in limpet grazing activity and microalgal abundance. Marine Ecology Progress Series, 211,193203.

Kim, M., Yang, E. C., \& Mansilla, A. (2004). Recent introduction of Polysiphonia morrowii (Ceramiales, Rhodophyta) to Punta Arenas, Chile. Botánica marina, 47,389-394.

Kiirikki, M. (1996). Experimental evidence that Fucus vesiculosus (Phaeophyta) controls filamentous algae by means of the whiplash effect. European Journal of Phycology, 31, 61-66.

Kim, D. (2001). Seasonality of marine algae and grazers of an antarctic rocky intertidal, with emphasis on the role of the limpet Nacella concinna strebel (Gastropoda: Patellidae). Berichte zur Polar und Meeresforschung, 397, 1-136.

Klöser, H., Quartino M. L., \& Wiencke, C. (1996). Distribution of macroalgae, and macroalgal communities in gradients of physical conditions in Potter Cove, King George Island, Antarctica. Hydrobiologia, 333, 1-17.

Lancellotti, D., \& Vásquez, J. (2000). Zoogeografía de macroinvertebrados bentónicos de la costa de chile: contribución para la conservación marina. Revista Chilena de Historia Natural, 73, 99-129.

Lewis, J. R. (1964). The Ecology of Rocky Shores. English Universities Press, London. XII, 323 pp. Illus. 42s.

Lüder, U. H., Knoetzel, J., \& Wiencke, C.(2001). Acclimation of photosynthesis and pigments to seasonally changing light conditions in the endemic Antarctic red macroalga Palmaria decipiens. Polar Biology, 24, 598-603.

Lüder, U. H. (2003). Acclimation of the photosynthetic apparatus of the endemic Antarctic red macroalga Palmaria decipiens to seasonally changing light conditions. Ber Polarforsch Meeresforsch, 469,141 pp.

Magurran, A. (2004). Measuring biological diversity. Blackwell, malden.
Magurran, A. (1988). Ecological diversity and its measurement. Princeton university press, new jersey.

Mansilla, A., Palacios, M., Navarro, N., \& Ávila, M. (2008). Utilization of agricultural fertilizers in the culture of Gigartina skottsbergii (Rhodophyta, Gigartinales). Journal of Applied Phycology, 20, 889-896.

Mansilla, A., Ávila, M., Cáceres, J., Palacios, M., Navarro, N., Cañete, I., \& Oyarzún S. (2009). Diagnóstico bases biológicas explotación sustentable Macrocystis pyrifera, (Huiro), XII región código bip $n^{\circ}$ 30060262-0. Gobierno regional de magallanes y antártica chilena. Informe de proyecto, Universidad de Magallanes, Chile.

Mansilla, A., Ávila, M., Yokoya, N. (2012). Current knowledge on biotechnological interesting seaweeds from the Magellan Region, Chile. Revista Brasileira Farmacognosia, 22(4), 760-767.

Mansilla A., Ávila, M., Ramírez, M. E., Rodriguez, J. P, Rosenfeld, S, Ojeda, J., \& Marambio, J. (2013). Macroalgas marinas bentónicas del submareal somero de la ecorregión subantártica de Magallanes, Chile. Anales Instituto Patagonia, 41(2),49-62.

Mendoza, M. L. \& Nizovoy, A. (2000). Géneros de Macroalgas marinas de la Argentina, fundamentalmente de Tierra del Fuego. Editado por el Poder Legislativo de la Provincia de Tierra del Fuego, Antártida e Islas del Atlántico Sur. Argentina.

Moreno, C. E., \& Halffter, G. (2000). Assessing the completeness of bat biodiversity inventories using species accumulation curves. Journal of Applied Ecology, 37, 149-158.

Murray, S., \& Horn M. (1989). Seasonal dynamics of macrophyte populations from an eastern North Pacific rocky-interdital habitat. Botánica Marina, 32, 457-473.

Murray, N., Weisberg, S., Raimondi, P., Ambrose, R., Bell, C., Blanchette, C., Burnaford, J., ...\&Smith, J. (2016). Evaluating ecological states of rocky intertidal communities: a best professional judgment exercise. Ecological Indicators, 60, 802-814.

Ojeda, F., \& Santelices, B. (1984). Invertebrate communities in holdfasts of the kelp 
Macrocystis pyrifera from southern Chile. Marine Ecology Progress Series, 16, 6573.

Ojeda, J. (2013). Dinámica estacional de macroalgas y moluscos intermareales y su relación con el conocimiento tradicional ecológico yagán, en canales subantárticos del cabo de hornos: una aproximación biocultural desde la filosofía ambiental de campo. Tesis de post-grado, Facultad de Ciencias, Programa de Magister en Ciencias Universidad de Magallanes, Departamento de Ciencias, y Recursos Naturales, Universidad de Magallanes, Punta Arenas, Chile.

Ramírez, M. E., \& Santelices, B. (1991). Catálogo de las algas marinas bentónicas de la costa del pacífico temperado de sudamérica. Monografías Biológicas 5. Pontificia Universidad Católica de Chile. Santiago, chile.

Ramírez, M. E. (1995). Recolección y colecciones científicas de macroalgas marinas. En E. K. Alveal (Eds.), Manual de Métodos Ficológicos (pp. 417-428). Concepción, Chile: Universidad de Concepción.

Ramírez, M. E. (2010). Flora marina Bentónica de la región austral de Sudamérica y la Antártica. Anales del Instituto de la Patagonia, 38(1),57-71.

Rilov, G., \& Schiel, D. R. (2011). Community Regulation: The Relative Importance of Recruitment and Predation Intensity of an Intertidal Community Dominant in a Seascape Context. PLoS ONE, 6(8), 23958.

Ríos, C., Arntz, W., Gerdes, D., Mutschke, E., \& Montiel, A. (2007). Spatial and temporal variability of the benthic assemblages associated to the holdfasts of the kelp Macrocystis pyrifera in the Straits of Magellan, Chile. Polar Biology, 31,89-100.

Ríos, C., \& Mutschke, E. (1999). Community structure of intertidal boulder-cobble fields in the Straits of Magellan, Chile. Scientia Marina, 63(1), 193-201.

Reaugh-Flower, K., Branch, G., Harris, J., McQuaid, C., Currie, B., Dye, A., \& Robertson, B. (2011). Scale-dependent patterns and processes of intertidal mussel recruitment around southern Africa. Marine Ecology Progress Series, 434, 101-119.

Romo, H., Ávila, M., \& Candía, A. (2001). Manual de técnicas de cultivo y repoblación de "Luga Roja" (Gigartina skottsbergii). Proyecto FONDEF D97I1064 y D00I1109. Universidad de Concepción - IFOP, Chile.

Rosenfeld, S., Ojeda, j., Hune, M., Mansilla, A., \& Contador, T. (2014). Egg masses of the Patagonian squid Doryteuthis (Amerigo) gahi attached to giant kelp (Macrocystis pyrifera) in the sub-Antarctic ecoregion. Polar Research, 33, 21636.

Rosenfeld, S., Aldea, C., Mansilla, A., Marambio, J., \& Ojeda, J. (2015). Richness, systematics, and distributions of molluscs associated with the macroalga Gigartina skottsbergii in the Strait of Magellan: a biogeographic affinity study. Zookeys, 519, 49-100.

Skottsberg, C. (1941). Communities of marine algae in subantarctic and antarctic waters. Kongliga Suenska Vetenskap Akademiens Handlingar Tredje Serien, 19(4),1-92.

Soberón, J., \& Llorente, J. (1993). The use of the species accumulation functions for the prediction of species richness. Conservation Biology, 7, 480-488.

Soto, E., Báez, P., Ramírez, M.E., Letelier, S., Naretto, J., \& Rebolledo, A. (2012). Biotopos Marinos intermareales entre Canal Trinidad y Canal Smyth, Sur de Chile. Revista de Biología Marina y Oceanografía, 47(2), 177-191.

Soto, J. (1991). Aportación al estudio sobre la fenología reproductora de las algas pardas y verdes del litoral del sureste de España. Acta Botánica Malacitana, 16(2), 317-323.

Svendsen, H., Beszczynska-Moller, A., Hagen, J., Lefauconnier, B., Tverberg, V., Gerland, S., Borre, J.,...\& Dallman, W. (2002). The physical environment of KongsfjordenKrossfjorden an Arcticfjord system in Svalbard. Polar Research, 21(1), 133-166.

Thompson, R., Roberts, M., Norton, T., \& Hawkins, S. (2000). Feast or famine for intertidal grazing molluscs: a mis-match between seasonal variations in grazing intensity and the abundance of microbial 
resources. Hydrobiología, 440(1),357-367. Underwood, A. J. (2004). Landing on one's foot: small-scale topographic features of habitat and the dispersion of juvenile intertidal gastropods. Marine Ecology Progress Series, 268, 173e182.

Valdenegro, C., \& Silva, N. (2003). Caracterización oceanográfica física y química de la zona de canales y fiordos australes de chile entre el estrecho de Magallanes y Cabo de Hornos (cimar 3 fiordos). Ciencia y Tecnología del Mar, 26(2), 19-60.

Walther, B., \& Moore, J., (2005). The concepts of bias, precision, and accuracy, and their use in testing the performance of species richness estimators, with a literature review of estimator performance. Ecography, 28, 1-15.

Weykam G., Thomas D., \& Wiencke C. (1997). Growth and photosynthesis of the Antarctic red algae Palmaria decipiens (Palmariales) and Iridaea cordata (Gigartinales) during and following extended periods of darkness. Phycologia, 36,395-405.

Wiencke, C., \& Clayton, M. (2002). Antarctic Seaweeds. in: (j. w. wagele, ed.). Synopsis of the antarctic benthos. a.r.g. Antner Verlag $\mathrm{kg}$. Ruggell/ Lichtensteien.

Wulff, A., Iken, K., Quartino, L., Al-Handal, A., Wiencke C., \& Clayton, M. (2009). Biodiversity, biogeography and zonation of benthic micro- and macroalgae in the Arctic and Antarctic. Botánica Marina, 52, 491507.

Yodzis, P. (1993). Environment and trophodiversity. in: r.e. rickleffs \& d. schluter (eds.), species diversity in ecological communities, historical and geographical perspectives. University. Chicago Press, Chicago, Illinois. 